\title{
SOME ORTHOGONALITY EQUATION WITH TWO FUNCTIONS
}

\author{
RADOSŁAW ŁUKASIK
}

Received 07 September, 2016

Abstract. The aim of this paper is to describe the solution $(f, g)$ of the equation

$$
\langle f(x) \mid f(y)\rangle=\langle g(x) \mid y\rangle, x, y \in X,
$$

where $f: X \rightarrow Y, g: X \rightarrow X, X, Y$ are inner product spaces over the same field $\mathbb{K} \in\{\mathbb{R}, \mathbb{C}\}$.

2010 Mathematics Subject Classification: 39B52; 47A05; 47A62

Keywords: orthogonality equation, Hilbert space, bounded linear operator, self-adjoint operator

\section{INTRODUCTION}

Throughout this paper $X, Y$ are Hilbert spaces over the same field $\mathbb{K} \in\{\mathbb{R}, \mathbb{C}\},\langle\cdot \mid \cdot\rangle$ denotes the inner product and $\|\cdot\|$ the norm associated with it. We shall not distinguish between the symbols used for $X$ and $Y, D(T)$ denotes the domain of the operator $T$.

It is known that $h: X \rightarrow Y$ is a solution of the orthogonality equation:

$$
\langle h(x) \mid h(y)\rangle=\langle x \mid y\rangle, x, y \in X
$$

if and only if $h$ is a linear isometry (see, e.g. [6, Lemma 2.1.1 and the following Remark]).

J. Chmieliński [3] studied the generalized orthogonality equation

$$
\langle f(x) \mid g(y)\rangle=\langle x \mid y\rangle, x, y \in X,
$$

with two unknown functions $f, g: X \rightarrow Y$. The form of solutions of the above equation was presented by R. Łukasik and P. Wójcik [5], R. Łukasik [4].

Also in the paper of M. M. Sadr [8] we can find the Banach-orthogonality equation

$$
\langle f(x) \mid g(\alpha)\rangle=\langle x \mid \alpha\rangle, x \in E, \alpha \in E^{*},
$$

where $f: E \rightarrow F, g: E^{*} \rightarrow F^{*}, E, F$ are Banach spaces, $F$ is reflexive.

In this paper we take a different approach. Instead take two different functions on the left side of (1.1), we change only the right side of (1.1).

Now we recall some facts from the theory of adjoint operators. 
Definition 1. Let $D(f)$ be a linear subspace of $X, f: D(f) \rightarrow Y$ be a linear operator. Let further

$$
D\left(f^{*}\right)=\left\{y \in Y: \bigvee_{z \in X} \bigwedge_{x \in D(f)}\langle f(x) \mid y\rangle=\langle x \mid z\rangle\right\} .
$$

A function $f^{*}: D\left(f^{*}\right) \rightarrow X$ is called an adjoint operator (to $f$ ) if and only if

$$
\langle f(x) \mid y\rangle=\left\langle x \mid f^{*}(y)\right\rangle, x \in D(f), y \in D\left(f^{*}\right) \text {. }
$$

Remark 1 (see [1, §39]). Let $D(f)$ be a dense linear subspace of $X, f: D(f) \rightarrow Y$ be a linear operator. Then the adjoint operator exists and it is unique. Moreover $D\left(f^{*}\right)$ is a linear subspace of $Y, f^{*}$ is a linear operator and

$$
\operatorname{ker} f^{*}=(\operatorname{im} f)^{\perp}, \quad \operatorname{clim} f=\left(\operatorname{ker} f^{*}\right)^{\perp} .
$$

Definition 2. Let $D(f)$ be a linear subspace of $X$. A linear operator $f: D(f) \rightarrow$ $Y$ is called bounded if and only if there exists $M>0$ such that

$$
\|f(x)\| \leq M\|x\|, x \in D(f) .
$$

Remark 2 (see [1, §19]). Let $D(f)$ be a dense linear subspace of $X, f: D(f) \rightarrow Y$ be a linear and bounded operator. Then $f$ can be uniquely extended to a linear and bounded operator on $X$.

Definition 3. Let $D(g)$ be a dense linear subspace of $X$. A function $g: D(g) \rightarrow X$ is called a symmetric operator if and only if

$$
\langle g(x) \mid y\rangle=\langle x \mid g(y)\rangle, x, y \in D(g) .
$$

Definition 4. Let $D(g)$ be a dense linear subspace of $X$. A function $g: D(g) \rightarrow X$ is called a self-adjoint operator if and only if $D\left(g^{*}\right)=D(g)$ and $g^{*}=g$.

Remark 3. For bounded operators definitions of the symmetric operator and the self-adjoint operator are equivalent.

Definition 5. Let $D(g)$ be a dense linear subspace of $X$. A linear operator $g: D(g) \rightarrow$ $X$ is called positive if and only if

$$
\langle g(x) \mid x\rangle \geq 0, x \in D(g) .
$$

Theorem 1 (see [7, §104] and [2]). Let $g: X \rightarrow X$ be a self-adjoint and positive operator. Then there exists a unique self-adjoint and positive operator $T: X \rightarrow X$ such that $g=T \circ T$.

Because every symmetric and positive operator can be extended to a self-adjoint operator we have the following result.

Theorem 2. Let $D(g)$ be a dense linear subspace of $X, g: D(g) \rightarrow X$ be a symmetric and positive operator. Then there exists a symmetric and positive operator $h: D(h) \rightarrow X$ such that $D(g) \subset D(h)$ and $g=h \circ h$. 
Definition 6. Let $D(T)$ be a dense linear subspace of $X$. A linear operator $T: D(T) \rightarrow Y$ is called a linear isometry if and only if

$$
\|T(x)\|=\|x\|, x \in D(T) .
$$

Remark 4 (see Remark 2). Let $D(T)$ be a dense linear subspace of $X$. Let $T: D(T) \rightarrow Y$ be a linear isometry. Then $T$ can be uniquely extended to a linear isometry from $X$ to $Y$.

Remark 5. Let $D(T)$ be a dense linear subspace of $X$. Let $T: D(T) \rightarrow Y$ be a linear isometry. Then

$$
\langle T(x) \mid T(y)\rangle=\langle x \mid y\rangle, x, y \in D(T) .
$$

We would like to divide our consideration into two cases: $f, g$ are bounded or unbounded. In view of Lemma 1 we do not have other cases.

\section{MAIN RESUlT FOR BOUNDED OPERATORS}

Because the domain of a bounded operator can be extended to the whole space, we assume in this section that operators are defined on the whole $X$.

First we describe properties of solutions of equation (2.1).

Theorem 3. Let $f: X \rightarrow Y, g: X \rightarrow X$ satisfy the equation

$$
\langle f(x) \mid f(y)\rangle=\langle g(x) \mid y\rangle, x, y \in X .
$$

Then $f$ is linear and bounded, $g$ is a self-adjoint and positive operator, $\operatorname{ker} f=\operatorname{ker} g$.

Proof. We observe that

$$
\langle g(x) \mid y\rangle=\langle f(x) \mid f(y)\rangle=\overline{\langle f(y) \mid f(x)\rangle}=\overline{\langle g(y) \mid x\rangle}=\langle x \mid g(y)\rangle, x, y \in X .
$$

We have also

$$
\langle g(x) \mid x\rangle=\langle f(x) \mid f(x)\rangle \geq 0, x \in X .
$$

Hence $g$ is a self-adjoint and positive operator. In particular $g$ is continuous. Since

$$
\begin{aligned}
\langle f(z) \mid f(\alpha x+y)-\alpha f(x)-f(y)\rangle & =\langle g(z) \mid \alpha x+y\rangle-\alpha\langle g(z) \mid x\rangle-\langle g(z) \mid y\rangle \\
& =0, x, y, z \in X, \alpha \in \mathbb{K},
\end{aligned}
$$

then $f(\alpha x+y)-\alpha f(x)-f(y) \in \operatorname{im} f^{\perp}, x, y \in X, \alpha \in \mathbb{K}$, so $f$ is linear. We notice that

$$
\|f(x)\|^{2}=\langle f(x) \mid f(x)\rangle=\langle g(x) \mid x\rangle \leq\|g(x)\| \cdot\|x\| \leq\|g\| \cdot\|x\|^{2}, x \in X,
$$

which means that $f$ is bounded.

Finally, for $x \in X$ we have

$$
x \in \operatorname{ker} g \Longleftrightarrow \bigwedge_{y \in X}\langle g(x) \mid y\rangle=0 \Longleftrightarrow \bigwedge_{y \in X}\langle f(x) \mid f(y)\rangle=0 \Longleftrightarrow x \in \operatorname{ker} f .
$$


Theorem 4. Let $f: X \rightarrow Y$ be linear and bounded. Then there exists a unique function $g: X \rightarrow X$ such that $f$ and $g$ satisfy equation (2.1). Moreover $g=f^{*} \circ f$.

Proof. Let $g=f^{*} \circ f$. Hence

$$
\langle f(x) \mid f(y)\rangle=\left\langle f^{*}(f(x)) \mid y\right\rangle=\langle g(x) \mid y\rangle, x, y \in X .
$$

Assume that there exists $h: X \rightarrow X$ such that

$$
\langle f(x) \mid f(y)\rangle=\langle h(x) \mid y\rangle, x, y \in X .
$$

Hence

$$
\langle g(x)-h(x) \mid y\rangle=\langle f(x) \mid f(y)\rangle-\langle f(x) \mid f(y)\rangle=0, x, y \in X,
$$

so $h=g$.

Theorem 5. Let $g: X \rightarrow X$ be a self-adjoint and positive operator, $\operatorname{im} g$ be isometric with some subspace of $Y$. Then there exists a function $f: X \rightarrow Y$ such that $f$ and $g$ satisfy equation (2.1).

Proof. Let $X_{0}=\operatorname{clim} g, T: X_{0} \rightarrow Y$ be a linear isometry (as an extension of a linear isometry from $\operatorname{im} g$ to $Y$ ). In view of Theorem 1 there exists a self-adjoint operator $h: X \rightarrow X$ such that $g=h^{2}$. It is easy to see that $\operatorname{ker} h=\operatorname{ker} g=X_{0}^{\perp}$ and hence $\operatorname{clim} h=\operatorname{clim} g=X_{0}$. We define function $f: X \rightarrow Y$ by the formula

$$
f(x)=T(h(x)), x \in X \text {. }
$$

Then

$$
\begin{aligned}
\langle f(x) \mid f(y)\rangle & =\langle T(h(x)) \mid T(h(y))\rangle=\langle h(x) \mid h(y)\rangle \\
& =\left\langle h^{2}(x) \mid y\right\rangle=\langle g(x) \mid y\rangle, x, y \in X .
\end{aligned}
$$

In the above theorem the assumption that im $g$ is isometric with some subspace of $Y$ is necessary. If the space $Y$ is too small, then this theorem does not hold, which shows the below example.

Example 1 . Let $\mathbb{K}=\mathbb{R}=Y, X=\mathbb{R}^{2}, g=I d_{X}$. Suppose that there exists $f: X \rightarrow$ $Y$ such that (2.1) holds. Then

$$
f\left(x_{1}, x_{2}\right) \cdot f\left(y_{1}, y_{2}\right)=x_{1} y_{1}+x_{2} y_{2}, x_{1}, x_{2}, y_{1}, y_{2} \in \mathbb{R} \text {. }
$$

Hence

$$
\begin{aligned}
f(1,0)^{2} & =1, \\
f(0,1)^{2} & =1, \\
f(1,0) \cdot f(0,1) & =0,
\end{aligned}
$$

which is impossible. 


\section{MAIN RESULT FOR UNBOUNDED OPERATORS}

Theorem 6. Let $D$ be a dense linear subspace of $X, f: D \rightarrow Y, g: D \rightarrow X$ satisfy the equation

$$
\langle f(x) \mid f(y)\rangle=\langle g(x) \mid y\rangle, x, y \in D .
$$

Then $f$ is linear, $\operatorname{im} f \subset D\left(f^{*}\right), g$ is a symmetric and positive operator, $\operatorname{ker} f=$ ker $g$.

Proof. Since

$$
\begin{aligned}
\langle f(z) \mid f(\alpha x+y)-\alpha f(x)-f(y)\rangle & =\langle g(z) \mid \alpha x+y\rangle-\alpha\langle g(z) \mid x\rangle-\langle g(z) \mid y\rangle \\
& =0, x, y, z \in D, \alpha \in \mathbb{K},
\end{aligned}
$$

then $f(\alpha x+y)-\alpha f(x)-f(y) \in \operatorname{im} f^{\perp}, x, y \in D, \alpha \in \mathbb{K}$, so $f$ is linear. Hence

$$
\begin{aligned}
\langle g(\alpha x+y)-\alpha g(x)-g(y) \mid z\rangle & =\langle f(\alpha x+y) \mid f(z)\rangle-\alpha\langle f(x) \mid f(z)\rangle-\langle f(y) \mid f(z)\rangle \\
& =0, x, y, z \in D, \alpha \in \mathbb{K},
\end{aligned}
$$

so $g$ is also linear.

We observe that

$$
\langle g(x) \mid y\rangle=\langle f(x) \mid f(y)\rangle=\overline{\langle f(y) \mid f(x)\rangle}=\overline{\langle g(y) \mid x\rangle}=\langle x \mid g(y)\rangle, x, y \in D .
$$

We have also

$$
\langle g(x) \mid x\rangle=\langle f(x) \mid f(x)\rangle \geq 0, x \in X .
$$

Hence $g$ is a symmetric and positive operator.

Let $z \in \operatorname{im} f$. Then $z=f(y)$ for some $y \in D$. We have

$$
\langle f(x) \mid z\rangle=\langle f(x) \mid f(y)\rangle=\langle g(x) \mid y\rangle=\langle x \mid g(y)\rangle, x \in D,
$$

so $z \in D\left(f^{*}\right)$.

Finally, for $x \in D$ we have

$$
x \in \operatorname{ker} g \Longleftrightarrow \bigwedge_{y \in X}\langle g(x) \mid y\rangle=0 \Longleftrightarrow \bigwedge_{y \in X}\langle f(x) \mid f(y)\rangle=0 \Longleftrightarrow x \in \operatorname{ker} f .
$$

Lemma 1. Let $f: D \rightarrow Y, g: D \rightarrow X$ satisfy the equation

$$
\langle f(x) \mid f(y)\rangle=\langle g(x) \mid y\rangle, x, y \in D .
$$

Then $f, g$ are linear and $f$ is bounded if and only if $g$ is bounded.

Proof. In view of the previous theorem $f$ is linear, $g$ is symmetric, so it is also linear.

$(\Rightarrow)$ Let $f$ be bounded. We have

$$
\begin{aligned}
|\langle g(x) \mid y\rangle| & =|\langle f(x) \mid f(y)\rangle| \leq\|f(x)\| \cdot\|f(y)\| \\
& \leq\|f\|^{2} \cdot\|x\| \cdot\|y\|, x, y \in D .
\end{aligned}
$$


Fix $x \in D$. Let $\left(y_{n}\right)_{n \in \mathbb{N}} \subset D$ be a sequence convergent to $g(x)$. Hence

$$
\begin{aligned}
\|g(x)\|^{2}=|\langle g(x) \mid g(x)\rangle| & =\lim _{n \rightarrow \infty}\left|\left\langle g(x) \mid y_{n}\right\rangle\right| \leq \lim _{n \rightarrow \infty}\|f\|^{2} \cdot\|x\| \cdot\left\|y_{n}\right\| \\
& =\|f\|^{2} \cdot\|x\| \cdot\|g(x)\|,
\end{aligned}
$$

which means that $g$ is bounded.

$(\Leftarrow)$ Let $g$ be bounded. We have

$$
\begin{aligned}
\|f(x)\|^{2} & =\langle f(x) \mid f(x)\rangle=\langle g(x) \mid x\rangle \leq\|g(x)\| \cdot\|x\| \\
& \leq\|g\| \cdot\|x\|^{2}, x \in D,
\end{aligned}
$$

so $f$ is bounded.

Theorem 7. Let $D$ be a dense linear subspace of $X, f: D \rightarrow Y$ be linear and $\operatorname{im} f \subset D\left(f^{*}\right)$. Then there exists a unique function $g: D \rightarrow X$ such that $f$ and $g$ satisfy equation (3.1). Moreover $g=f^{*} \circ f$.

Proof. Because im $f \subset D\left(f^{*}\right)$, then $g=f^{*} \circ f$ is well-defined. Hence

$$
\langle f(x) \mid f(y)\rangle=\left\langle f^{*}(f(x)) \mid y\right\rangle=\langle g(x) \mid y\rangle, x, y \in D .
$$

Assume that there exists $h: D \rightarrow X$ such that

$$
\langle f(x) \mid f(y)\rangle=\langle h(x) \mid y\rangle, x, y \in D .
$$

Hence

$$
\langle g(x)-h(x) \mid y\rangle=\langle f(x) \mid f(y)\rangle-\langle f(x) \mid f(y)\rangle=0, x, y \in D,
$$

so $h=g$.

Theorem 8. Let $D$ be a dense linear subspace of $X, g: D \rightarrow X$ be a symmetric and positive operator, $\mathrm{im} g$ be isometric with some subspace of $Y$. Then there exists a function $f: D \rightarrow Y$ such that $f$ and $g$ satisfy equation (3.1).

Proof. Let $X_{0}=\operatorname{clim} g, T: X_{0} \rightarrow Y$ be a linear isometry (as an extension of a linear isometry from $\operatorname{im} g$ to $Y$ ). In view of Theorem 2 there exists a symmetric operator $h: D(h) \rightarrow X$ such that $D \subset D(h)$ and $g=h^{2}$. It is easy to see that $\operatorname{cl} X_{0}^{\perp}=\operatorname{cl} \operatorname{ker} g \subset \operatorname{cl} \operatorname{ker} h$ and hence $\operatorname{clim} h=(\operatorname{ker} h)^{\perp} \subset(\operatorname{ker} g)^{\perp}=\operatorname{clim} g=X_{0}$. We define a function $f: D \rightarrow Y$ by the formula

$$
f(x)=T(h(x)), x \in D .
$$

Then

$$
\begin{aligned}
\langle f(x) \mid f(y)\rangle & =\langle T(h(x)) \mid T(h(y))\rangle=\langle h(x) \mid h(y)\rangle \\
& =\left\langle h^{2}(x) \mid y\right\rangle=\langle g(x) \mid y\rangle, x, y \in D .
\end{aligned}
$$


The following example shows that we do not have uniqueness of $f$ in Theorems 5 and 8 .

Example 2. Let $f, g, h: \mathbb{K}^{3} \rightarrow \mathbb{K}^{3}$ be maps given by formulas

$$
\begin{aligned}
f\left(x_{1}, x_{2}, x_{3}\right) & =\left(x_{2}, x_{1}, x_{3}\right), x_{1}, x_{2}, x_{3} \in \mathbb{K}, \\
h\left(x_{1}, x_{2}, x_{3}\right) & =\left(x_{1}, x_{3}, x_{2}\right), x_{1}, x_{2}, x_{3} \in \mathbb{K}, \\
g & =I d_{\mathbb{K}^{3}} .
\end{aligned}
$$

It is easy to see that $f^{2}=h^{2}=I d_{\mathbb{K}^{3}}$ and $f^{*}=f, h^{*}=h$. Hence

$$
\langle f(x) \mid f(y)\rangle=\langle h(x) \mid h(y)\rangle=\langle x \mid y\rangle=\langle g(x) \mid y\rangle, x, y \in \mathbb{K}^{3} .
$$

But we can also say something about the family of functions $f$ which satisfies equation (3.1) with the same $g$.

Theorem 9. Let $D$ be a dense linear subspace of $X, f: D \rightarrow Y, g: D \rightarrow X$ satisfy equation (3.1). Then $h: D \rightarrow Y$ satisfies the equation

$$
\langle h(x) \mid h(y)\rangle=\langle g(x) \mid y\rangle, x, y \in D,
$$

if and only if there exists a linear isometry $I: \operatorname{im} f \rightarrow Y$ such that $h=I \circ f$.

Proof. $(\Longrightarrow)$ Let $h: D \rightarrow Y$ satisfy equation (3.2). We know that $f, h$ are linear. We define $I: \operatorname{im} f \rightarrow Y$ by the formula

$$
I(f(x))=h(x), x \in D .
$$

We observe that

$$
\begin{aligned}
f(x)=f(y) & \Longleftrightarrow x-y \in \operatorname{ker} f \Longleftrightarrow x-y \in \operatorname{ker} g \\
& \Longleftrightarrow x-y \in \operatorname{ker} h \Longleftrightarrow h(x)=h(y), x, y \in D,
\end{aligned}
$$

so $I$ is well-defined. We have also

$$
\begin{aligned}
I(\alpha f(x)+f(y)) & =I(f(\alpha x+y))=h(\alpha x+y)=\alpha h(x)+h(y) \\
& =\alpha I(f(x))+I(f(y)), x, y \in D, \alpha \in \mathbb{K}
\end{aligned}
$$

and

$$
\|I(f(x))\|^{2}=\|h(x)\|^{2}=\langle g(x) \mid x\rangle=\|f(x)\|^{2}, x \in D .
$$

Hence $I$ is a linear isometry and $h=I \circ f$.

$(\Longleftarrow)$ Let $I: \operatorname{im} f \rightarrow Y$ be a linear isometry, $h=I \circ f$. Then

$$
\langle h(x) \mid h(y)\rangle=\langle I(f(x)) \mid I(f(y))\rangle=\langle f(x) \mid f(y)\rangle=\langle g(x) \mid y\rangle, x, y \in D .
$$




\section{REFERENCES}

[1] N. I. Akhiezer and I. M. Glazman, Theory of linear operators in Hilbert space. New York: Dover Publications Inc., 1993.

[2] S. J. Bernau, "The square root of positive self-adjoint operator," J. Austral. Math. Soc., vol. 8, no. 1, pp. 17-36, 1968, doi: 10.1017/S1446788700004560.

[3] J. Chmieliński, "Orthogonality equation with two unknown functions," Aequat. Math., vol. 90, no. 1, pp. 11-23, 2016, doi: 10.1007/s00010-015-0359-x.

[4] R. Łukasik, "A note on the orthogonality equation with two functions," Aequat. Math., vol. 90, no. 5, pp. 961-965, 2016, doi: 10.1007/s00010-016-0419-xx.

[5] R. Łukasik and P. Wójcik, "Decomposition of two functions in the orthogonality equation," Aequat. Math., vol. 90, no. 3, pp. 495-499, 2016, doi: 10.1007/s00010-015-0385-8.

[6] W. Mlak, Hilbert Spaces and Operator Theory. Boston: Kluwer Academic Publishers, 1991.

[7] F. Riesz and B. Sz.-Nagy, Functional Analysis. New York: Dover Publications Inc., 1990.

[8] M. M. Sadr, "Decomposition of functions between Banach spaces in the orthogonality equation," Aequat. Math., 2017, doi: 10.1007/s00010-017-0466-y.

Author's address

\section{Radosław Lukasik}

Institute of Mathematics, University of Silesia, 40-007 Katowice, ul. Bankowa 14, POLAND

E-mail address: rlukasik@math.us.edu.pl 